\title{
A Novel TPS Toolkit to Assess Correlation Between Transit Fluence Dosimetry and DVH Metrics for Adaptive Head and Neck Radiotherapy
}

George Antoniou ( $\sim$ george.antoniou@sa.gov.au )

SA Medical Imaging https://orcid.org/0000-0003-3639-6812

\section{Scott Penfold}

Royal Adelaide Hospital

\section{Research Article}

Keywords: Adaptive radiotherapy, Volumetric modulated arc therapy (VMAT), Transit dosimetry, RayStation scripting

Posted Date: May 25th, 2021

DOI: https://doi.org/10.21203/rs.3.rs-541098/v1

License: (c) (i) This work is licensed under a Creative Commons Attribution 4.0 International License.

Read Full License

Version of Record: A version of this preprint was published at Physical and Engineering Sciences in Medicine on August 31st, 2021. See the published version at https://doi.org/10.1007/s13246-021-010485 . 
1 A Novel TPS Toolkit to Assess Correlation between Transit Fluence Dosimetry

2

4

5 and DVH Metrics for Adaptive Head and Neck Radiotherapy

\author{
George Antoniou ${ }^{1,2}$ \\ ORCiD: 0000-0003-3639-6812
}

\title{
Scott N. Penfold ${ }^{1,3}$
}

ORCiD: 0000-0002-3422-9108

Corresponding Author: george.antoniou@adelaide.edu.au

${ }^{1}$ Department of Physics, The University of Adelaide, Adelaide, SA 5005, Australia

${ }^{2}$ Medical Physics Group, SA Medical Imaging, Adelaide, SA 5000, Australia

${ }^{3}$ Department of Radiation Oncology, Royal Adelaide Hospital, Adelaide, SA 5000, Australia

\section{Abstract}

Inter-fractional anatomical variations in head and neck $(\mathrm{H} \& \mathrm{~N})$ cancer patients can lead to clinically significant dosimetric changes. Adaptive re-planning should thus commence to negate any potential over-dosage to organsat-risk (OAR), as well as potential under-dosage to target lesions. The aim of this study is to explore the correlation between transit fluence, as measured at an electronic portal imaging device (EPID), and dose volume histogram (DVH) metrics to target and OAR structures in a simulated environment. Planning data of 8 patients that have previously undergone adaptive radiotherapy for head and neck cancer using volumetric modulated arc therapy (VMAT) at the Royal Adelaide Hospital were selected for this study. Through delivering the original treatment plan to both the planning and rescan CTs of these 8 patients, predicted electronic portal images (EPIs) and DVH metrics corresponding to each data set were extracted using a novel RayStation script. A weighted projection mask was developed for target and OAR structures through considering the intra-angle overlap between fluence and structure contours projected onto the EPIs. The correlation between change in transit fluence and planning target volume (PTV) D98 and spinal cord D0.03cc with and without the weighting mask applied was investigated. PTV D98 was strongly correlated with mean fluence percentage difference both with and without the weighting mask applied $\left(\mathrm{R}_{\text {Mask }}=0.69, \mathrm{R}_{\text {No Mask }}=0.79, \mathrm{~N}=14, \mathrm{p}<0.05\right)$, where spinal cord D0.03cc exhibited a weak correlation $\left(\mathrm{R}_{\text {Mask }}=0.35, \mathrm{R}_{\mathrm{No} \text { Mask }}=0.53, \mathrm{~N}=7, \mathrm{p}>0.05\right)$ however this result was not statistically significant. The simulation toolkit developed in this work provided a useful means to investigate the relationship between change in transit fluence and change in key dosimetric parameters for head and neck cancer patients.

Keywords Adaptive radiotherapy $\cdot$ Volumetric modulated arc therapy $($ VMAT) $\cdot$ Transit dosimetry $\cdot$ RayStation scripting 


\section{Declarations}

Funding - No funding was received for conducting this study.

Conflicts of interest/Competing interests - The authors have no relevant financial or non-financial interests to disclose.

Availability of data and material - Not applicable

Code availability - Not applicable

Ethics approval - Approval was obtained from the Central Adelaide Local Health Network Human Research Ethics Committee (CALHN HREC). The procedures used in this study adhere to the tenets of the Declaration of Helsinki.

Consent to participate - Each participant has given consent to participate, via the statement "I do approve the use of my radiation therapy records for retrospective medical auditing and / or research.

Consent for publication - Not applicable 


\subsection{Introduction}

Anatomical variations in head and neck $(\mathrm{H} \& \mathrm{~N})$ cancer patients can lead to clinically significant plan deterioration and adaptive radiotherapy (ART) may be required to restore an optimal dose distribution [1]. Past literature has explored the benefits of implementing ART workflows within a clinic for H\&N cancer and has mostly been focused on improving dosimetry in parotid glands, which is crucial when lowering risk of xerostomia [1], [2]. Monitoring the dose delivered to the spinal cord, via evaluation of $\mathrm{kV}$ cone-beam computed tomography (CBCT), has also shown to be beneficial in answering the golden question: when should we replan? [3]

Traditional methods to implement ART into the clinic are associated with a significant increase in the clinical workload to the radiotherapy department as a whole. As a result, there has been a recent push to develop an accessible and automated quantitative trigger for ART, with one common method being transit dosimetry with an electronic portal imaging device (EPID) [4]. Using relative EPID dosimetry, one can explore the gradual change in transit fluence over the course of treatment without necessarily needing to rely on the absolute precision and accuracy of the images themselves. Change in transit fluence is expected to be correlated with anatomical variations and patient setup errors, which has thus far been the main focus of research on this topic. Several groups have tried to correlate dosimetric impacts with change in transit fluence, where 2D relative gamma analysis is commonly utilised [5]-[8]. Through conducting gamma analysis on the transit EPID images acquired over the course of treatment, a variety of parameters can be extracted to help quantify change in dose to the patient.

Through utilising the mean gamma value, $\gamma_{\text {mean }}$, from a 2D 3\%/3mm relative gamma analysis test on whole electronic portal images (EPIs), Piron et al. [5] concluded that change in transit fluence could be used as a predictor for plan deterioration for $\mathrm{H} \& \mathrm{~N}$ cancer patients as a result of anatomical variations. Utilising the whole EPI, however, could result in misleading mean gamma values. If only a small area of pixels included large deviations, the EPI as a whole may still be similar to the baseline EPI and thus result in a score that is below the trigger threshold, ultimately decreasing sensitivity. By considering projections of regions of interest onto the EPIs, the sensitivity of the analysis may be improved.

By projecting planning target volume's (PTV's) onto EPIs obtained every fraction and correlating $\gamma_{\text {mean }}$ with dosimetric changes (V95\%), Piron et al. [6] found that projecting PTVs onto the EPIs and then conducting gamma analysis improved sensitivity to anatomical changes. However, projecting OARs onto the EPIs were not considered.

The same group then went on to establish an action threshold for H\&N ART, and proposed a threshold of $\gamma_{\text {mean }}>0.42$, as evaluated using the whole EPI [7]. By considering a dosimetric threshold of V100\% $<90 \%$ the group was able to analyse the sensitivity and specificity of the threshold proposed. Moreover, the group also explored the correlation between mean dosimetric differences of PTV and OARs with mean gamma values of the whole EPI for patients that did reach the action threshold, as well as patients that did not. A strong correlation between change in spinal cord dose and $\gamma_{\text {mean }}$ was not observed, likely due to the inherent nature of the gamma analysis test conducted on whole EPIs - being more sensitive in high dose regions, such as PTVs, than lower dose regions, such as OARs.

Lim et al. [9] explored the correlation between change in transit fluence, in a generalised rectangular region surrounding the neck, and volumetric change of a ROI $\left(\Delta V_{R O I}\right)$ spanning from the Condyloid process (jaw) to C6 of the spinal cord. Volumetric change, which is a good predictor for grade 2 xerostomia [10], was found to be strongly correlated with change in transit fluence $(R=-0.776, p<0.001)$. A $5 \%$ threshold in $\Delta V_{R O I}$ could be 
used as a trigger for ART, where the area under the receiver-operating characteristic curve (ROC) was determined to be 0.88 . This study did not investigate the potential improvement in sensitivity by projecting the ROI onto the EPID.

When utilising linac-measured EPIs for relative gamma analysis, one of the largest sources of systematic error in these types of studies include the accuracy of the first fraction EPI. The results of these studies all rely on the assumption that the patient anatomy at the time of the first fraction EPI is representative of the patient anatomy at the time of the planning CT (pCT). A poor baseline could be misleading and yield results with $\gamma_{\text {mean }}$ values significantly lower than actually representative of the change since $\mathrm{pCT}$. The research presented in this study differs from previous studies by predicting transit EPIs using an in-house script developed in the RayStation treatment planning system (TPS) by RaySearch Laboratories, rather than analysing linac-measured EPIs. The advantage of this approach is in the removal of any patient set-up errors, as well as any anatomical variation in the patient between obtaining the pCT and first fraction baseline EPI. Rather than using weekly CBCTs to obtain multiple EPIs over the course of treatment, this study will also only consider the pCT and rescan CT (rCT) to avoid any uncertainties associated with deformable image registration of the pCT's to CBCT's, or dose calculation uncertainty on CBCT. Considering these factors, the developed tool allows for the investigation of correlation between change in transit fluence and change in patient DVH metrics in a more controlled environment.

\subsection{Methods}

\subsection{Patient selection}

Human research ethics and research governance approval was obtained for the study. The radiotherapy datasets (treatment plans, planning CTs and RT structure sets) of eight patients previously having undergone adaptive radiotherapy for $\mathrm{H} \& \mathrm{~N}$ cancer at the RAH were collected and anonymized. Each patient consented to their data being used for research purposes and had at least 1 rescan CT acquired over the course of treatment.

\subsection{EPI Prediction in RayStation}

\subsubsection{EPID Model}

The EPID model developed in this work was generated to represent EPI's measured with a Varian TrueBeam aS1200 MV imager in Portal Dosimetry mode. Square field Portal Dosimetry images were collected to provide beam profiles and output factors with and without solid water in place and the EPID at $150 \mathrm{~cm}$ source to detector distance (SDD).

A simple EPID model was constructed in RayStation to model the Varian TrueBeam aS1200 MV imager operating in Portal Dosimetry mode. The model consisted of a $40 \mathrm{~cm} \times 40 \mathrm{~cm} \times 5 \mathrm{~cm}$ water slab atop a $40 \mathrm{~cm} \times 40 \mathrm{~cm} \times 4 \mathrm{~cm}$ lead slab. Dose planes were extracted at $3.6 \mathrm{~cm}$ depth in the water slab. The selected water slab thickness and extraction depth were guided by Varian Portal Dosimetry calibration settings. The thickness of the lead slab was selected based on a comparison of measured and computed output factors. Calculated EPIs simulated in RayStation at $150 \mathrm{~cm}$ SDD were first downscaled to $100 \mathrm{~cm}$ SDD, where flood field and beam profile corrections were applied, and subsequently scaled back to $150 \mathrm{~cm}$ SDD. This was done to mimic the major corrections applied in forming an EPI in portal dosimetry mode on the linac. 


\subsubsection{EPI Extraction}

The RayStation EPID template model was imported as regions of interest into the original treatment plan of a given patient within RayStation. By utilising a number of functions within the RayStation python scripting environment, an automated sequence of steps was programmed to rotate the EPID model around isocenter for each control point of a VMAT plan as outlined in Fig. 1. The EPID model was first positioned to have the $3.6 \mathrm{~cm}$ extraction depth positioned $50.0 \mathrm{~cm}$ below the origin. This was followed by rotation to a given control point, and subsequent translation to be centred at the isocenter. Once positioned appropriately, the dose for that VMAT control point was calculated. The dose delivered to the extraction plane within the EPID model was then stored into a 200 x 200 pixel array, with a pixel size of $2.0 \mathrm{~mm}$, and subsequently integrated over every control point of the VMAT plan. To help optimise the time required to extract the EPIs from $n$ beams, the dose from all beams at a given gantry angle was calculated, rather than rotating the model around the patient $n$ times. Thus, the generated image is a sum of all beams in a fraction, rather than per-beam images. Once the original treatment plan had been simulated using the $\mathrm{pCT}$, the process was repeated using the $\mathrm{rCT}$ to obtain a second EPI.
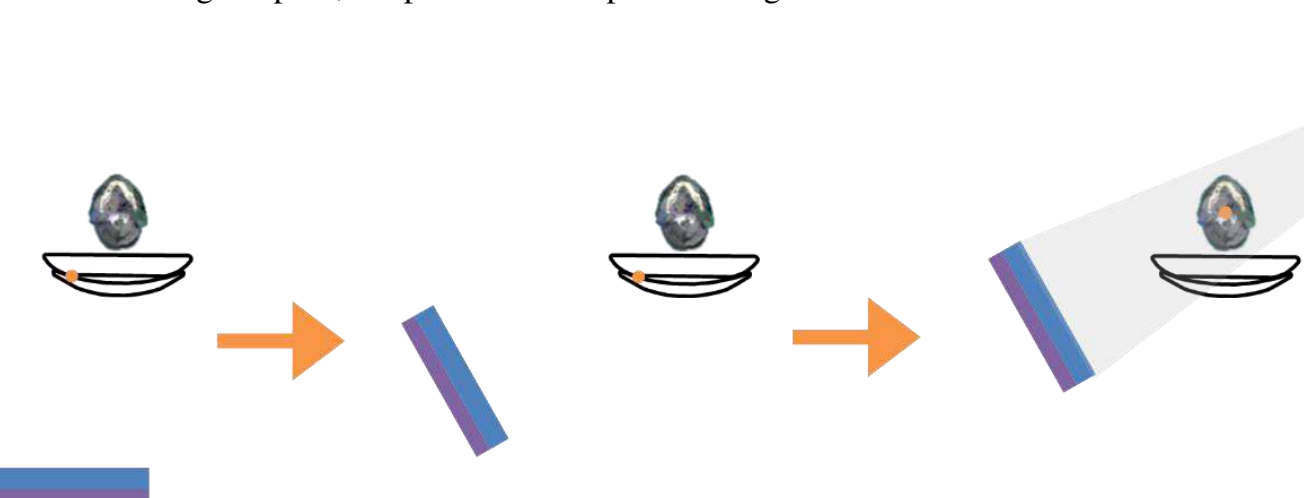

Fig. 1 EPID extraction process repeated along every control point for both the pCT and rCT. EPID first positioned $50.0 \mathrm{~cm}$ below origin, then rotated to a given control point angle, and translated to $50.0 \mathrm{~cm}$ from isocenter

\subsubsection{VMAT Script Validation}

To validate our model, a $20.0 \times 20.0 \times 10.0 \mathrm{~cm}$ thick solid water slab was positioned at $90.0 \mathrm{~cm}$ SSD on a Varian TrueBeam linac, with the EPID positioned at $150.0 \mathrm{~cm}$ SDD. A VMAT treatment plan was then delivered, and a baseline EPI was extracted. To simulate change in patient anatomy, $2.0 \mathrm{~cm}$ of the solid water slab was removed anteriorly and the same VMAT treatment plan was delivered to extract a second fraction EPI. The change between the second EPI and the baseline was then calculated as a 2D percentage difference map, relative to dose maximum of the baseline. This process was then repeated using the simulation toolkit developed in RayStation to obtain a predicted 2D percentage difference map.

\subsection{ROI Projection Mask}

Past research has shown that projecting ROI's onto the EPIs can lead to improved sensitivity in using change in transit fluence as a quantitative trigger for adaptive radiotherapy. The contours of the spinal cord and PTV's for each patient included in the study were thus extracted through utilising DICOM RT structure files. These contours could then be projected onto the extraction plane of the EPI with the use of modules within the dicompyler-core Python package [11]. A ROI projection mask was then created, taking the thickness of the structure in the beam'seye-view into consideration to weight the mask by the path length through the structure as shown in Fig. 2.a). 
In this work, the spinal cord was chosen as a significant ROI alongside the PTV's as plan adaption for H\&N patients at the Royal Adelaide Hospital is often triggered by the monitoring of spinal cord doses during on-course plan dosimetry assessment. However, the methods outlined in this work can still be applied to a variety of different structures, such as the parotid glands when considering toxicities such as xerostomia [9], [10]. If a plan contained multiple PTV volumes with different dose levels, each was treated as an independent ROI in the correlation analysis. The evaluation PTV contours (PTV_EVAL), as calculated via subtracting the higher dose PTV's from the lower dose PTV's, were utilised as these are the volumes that undergo DVH assessment during treatment planning.

To further evolve the ROI projection mask, one can consider the overlap between the open field of the MLC configuration and the projection of the structure of interest. Thus, the DICOM plan file was also used to extract these coordinates on the EPI to create a 'fluence projection' mask. EPI pixels that lay within the open field projection were given a value of 1 , and those outside the open field were given a value of 0 . The mask could then further be weighted by the monitor units (MU's) delivered to the open field region for a given control point through multiplying the mask through by the cumulative meterset value and beam MU's found in the DICOM RT plan file as shown in Fig. 2.b). The resulting mask could then be integrated along each control point to obtain a final intraangle projection mask as shown in Fig. 2.e), which highlights the regions within the EPI for which dose was delivered to a particular structure of interest, weighted by the structure's volume and MU's delivered.
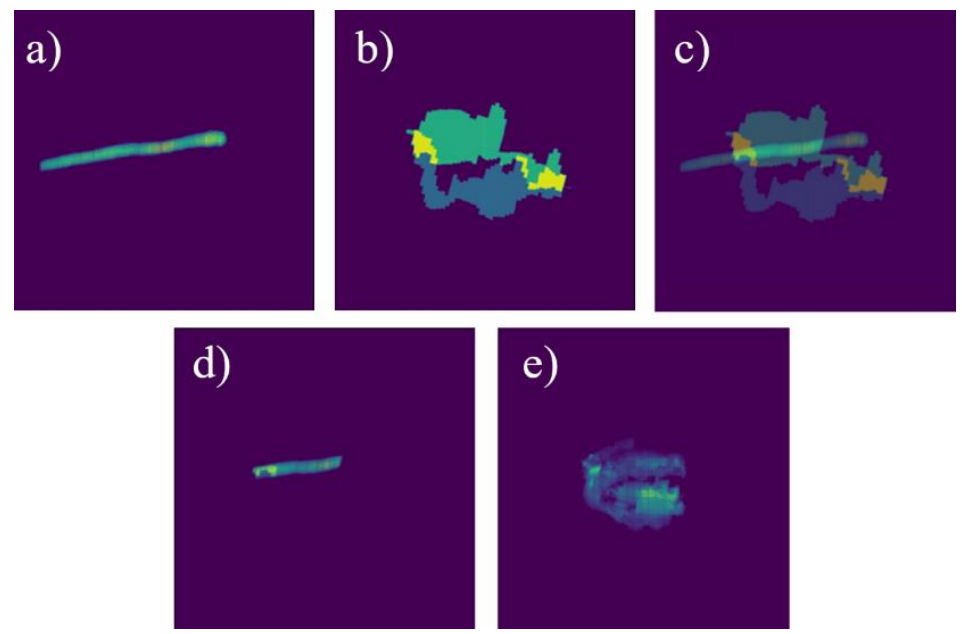

Fig. 2 Process used to derive an intra-angle ROI and fluence projection mask. For each control point, a) the structure projection mask and b) fluence projection is used to obtain d) the overlap between the two, as visualised by c). This process is repeated for each control point to achieve e) the final intra-angle ROI and fluence mask

\subsection{DVH Parameters}

Change in transit fluence alone cannot be used as a reliable trigger for adaptive radiotherapy without first understanding how this quantity relates to dosimetric differences within the patient. Current workflows at the Royal Adelaide Hospital involve an on-course dosimetry monitoring program to ensure target coverage does not diminish by a certain amount and that serial organs at risk, such as the spinal cord, do not exceed tolerance values. In this study DVH metrics considered for the spinal cord and PTV_EVAL structures relate to the near maximum and minimum doses received by the volumes, being the D0.03cc and D98 metric respectively. In this work, the change in these metrics over the two $\mathrm{CT}$ datasets (original planning $\mathrm{CT}$ and rescan $\mathrm{CT}$ ) was considered and any correlation between them and change in transit fluence was explored. 


\subsection{Correlation}

As previously mentioned, a correlation between change in transit fluence and change in D0.03cc or D98, for the spinal cord and PTV's respectively, would show that change in transit fluence can be used as a trigger for adaptive radiotherapy. There have been multiple approaches in quantifying this change in transit fluence to be used as a trigger for adaptive radiotherapy, where gamma parameters are commonly utilised such as $\gamma_{\text {max }}, \gamma_{\text {mean }}$, and $\gamma_{1 \%}$. One disadvantage of this approach is that gamma values are always positive and thus give no information regarding the direction of such change. A second fraction EPI that has received a greater amount of dose than the baseline may show similar corresponding gamma parameters to a second fraction EPI that has received less dose than the baseline. It was thus decided that the mean percentage difference between the two EPIs should be calculated, relative to the baseline, as this will also provide directional information. It should be noted, however, that signed percentage differences in a region of interest may cancel and lead to lower-than-expected mean values. Care should thus be taken when interpreting these values via first assessing the 2D percentage difference map.

A correlation between the change in D0.03cc and D98, for the spinal cord and PTV_EVAL's, and mean fluence percentage difference with and without the respective masks applied was then explored. Noting that with no mask applied, the entire 40x40 cm EPIs were utilised, whilst disregarding doses $<10 \%$ of local maximum. Statistical analysis was conducted through considering Pearson's correlation coefficient $(R)$, Spearman's rank correlation coefficient $(\rho)$, and a linear regression with a $95 \%$ confidence interval. The statistical significance of the correlation coefficients was also calculated for the given sample size, where the difference between the correlation with and without the projection masks applied was also considered to explore any improvements in sensitivity that the mask may supply.

\subsection{Results}

\subsection{EPID Model}

\subsubsection{Non-Transit Verification}

The EPID model used in this work was developed through consideration of beam profiles, output factors, and gamma analysis between predicted and measured EPIs. Fig. 3 shows beam profiles from linac-measured and RayStation calculated EPIs after delivering $2.0 \times 2.0,3.0 \times 3.0,4.0 \times 4.0,5.0 \times 5.0,10.0 \times 10.0$, and $20.0 \times 20.0 \mathrm{~cm}^{2}$ square fields with the EPID positioned at $150 \mathrm{~cm}$ SDD. The output factors of these square fields were also obtained, as shown in Fig. 4, showing percentage differences all less than $0.8 \%$.
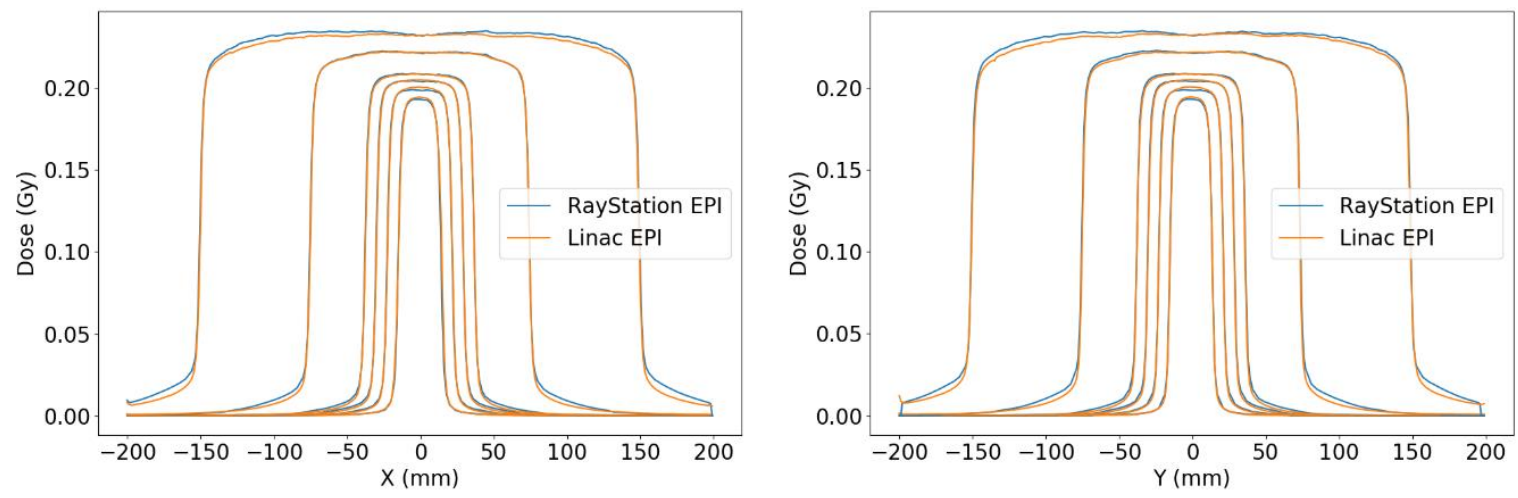

Fig. 3 True (Linac) and predicted (RayStation) non-transit beam profiles with EPID positioned at $150 \mathrm{~cm}$ 




Fig. 4 True (Linac) and calculated (RayStation) output factors from square fields, with percentage difference

\subsection{EPID Model Validation}

The EPID model developed was first validated through simulating change in patient anatomy via anteriorly removing $2.0 \mathrm{~cm}$ of a solid water slab between two deliveries of a VMAT plan. Changes in the linac-measured EPIs were compared to changes in the TPS-calculated EPIs through comparing percentage differences, $\Delta \phi_{\%}$, in beam profiles between the two exposures as shown in Fig. 5. It can be seen that majority of the error lies within the field, where \%difference in regions of high dose gradient between the predicted and measured changes adequately agree with one another. In the x-profiles shown, a maximum percentage difference of $7.2 \%$ and $7.7 \%$ was observed for the predicted and true changes in EPIs, respectively.
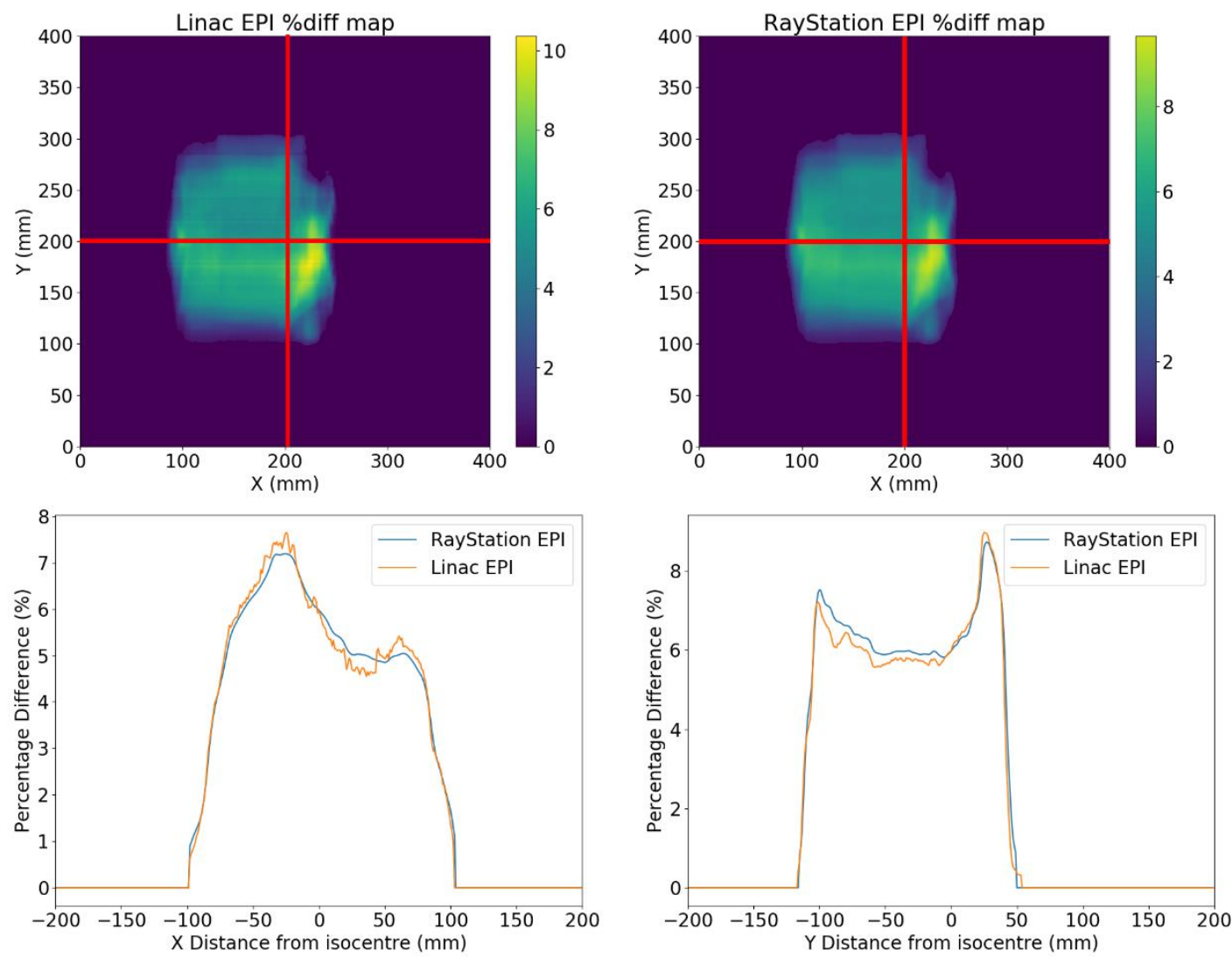

Fig. 5 True (Linac) and calculated (RayStation) 2D percentage difference maps used for model validation, with $\mathrm{x}$ and y-profiles 
Similarly, for the y-profiles, a maximum percentage difference of $8.7 \%$ and $9.0 \%$ were observed for the predicted and true change in EPIs. The mean percentage error between the true and predicted percentage differences was $1.49 \%$, ranging between a maximum of $17.4 \%$ and a minimum of $<0.01 \%$. The $2 \mathrm{D}$ percentage error map between the two percentage differences is shown in Fig. 6. The TPS simulation was thus considered as a good indicator of what one would measure on a linac MV imager in the corresponding situation.
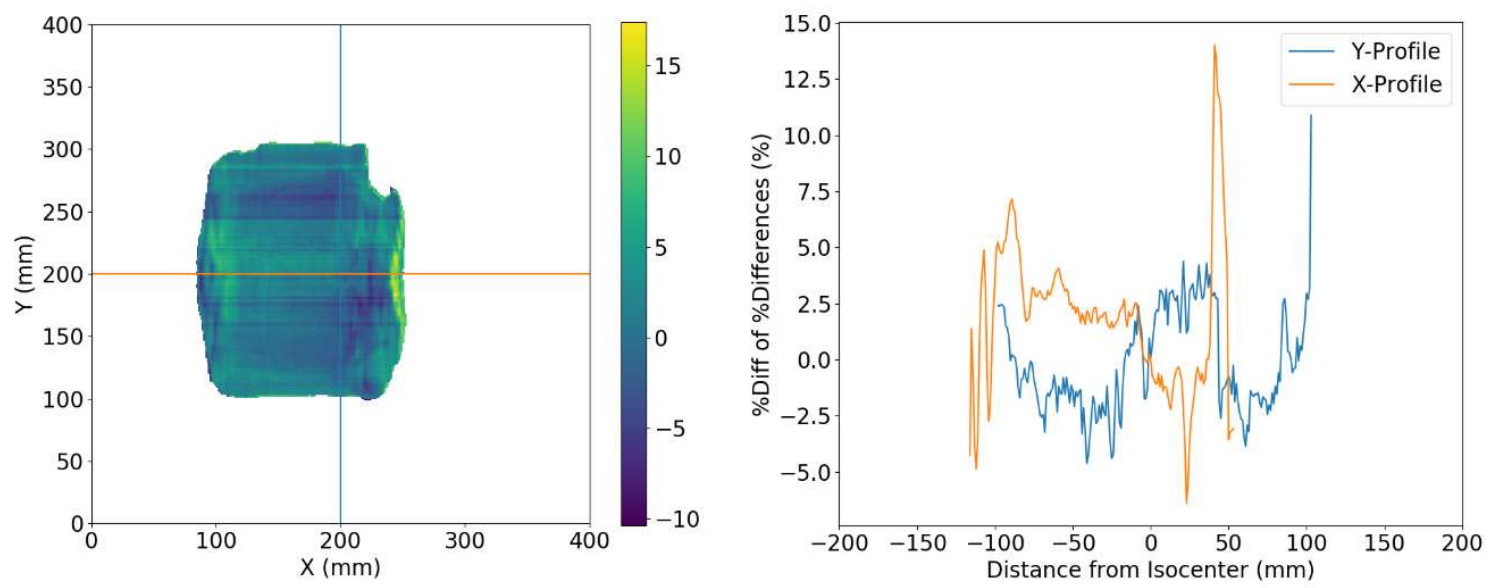

Fig. 6 2D percentage error map of the 2D percentage difference maps used for EPID model validation, with Xand Y-profiles

\subsection{Correlation of change in transit fluence and change in DVH metrics}

\subsubsection{No Weighting Mask Applied}

The correlation between change in transit fluence and change in DVH metrics for the 8 patient datasets was explored. D98 PTVs and D0.03cc for the spinal cord was first explored with no weighting masks applied - utilising the entire $40 \times 40 \mathrm{~cm}$ EPIs whilst disregarding doses $<10 \%$ of local maximum. Fig. 7.a) shows the relationship between mean transit fluence percentage difference and change in D98 for the PTVs, with a linear regression band fitted to a 95\% confidence interval. Pearson's and Spearman's correlation coefficients were determined to be 0.79 and 0.82 respectively, indicating a strong correlation exists. Similarly, Fig. 7.b) shows the relationship between change in $\mathrm{D} 0.03 \mathrm{cc}$ for the spinal cord and mean transit fluence percentage difference, also with a linear regression band fitted to a $95 \%$ confidence interval. Pearson's correlation coefficient of 0.53 indicates a moderate correlation exists between the two parameters, as well as Spearman's correlation coefficient of 0.32 .

\subsubsection{Weighting Masks Applied}

\section{ROI Projection Mask}

To observe the individual benefits of various contributions to the overall weighting mask applied, the effects of projecting the ROIs onto the EPIs were first considered. Fig. 7.c) and Fig. 7.d) show the correlation between change in D98 and D0.03cc with mean weighted fluence percentage difference, respectively. Pearson's and Spearman's correlation coefficients suggest that strong and moderate correlations exist for the PTVs and spinal cords, respectively, yielding values of 0.70 and 0.67 for the PTVs and 0.41 and 0.32 for the spinal cords. The slope of the linear regression fit for both the PTVs and spinal cords increase when utilising the ROI projection mask, in comparison to no weighting mask applied. This result suggests that the weighting mask applied increases the overall sensitivity of the fit, as expected. 


\section{Intra-angle ROI and Fluence Projection Mask}

The final level of complexity added to the weighting projection mask includes an intra-angle convolution between a fluence projection mask, through considering the MLC leaf configuration, and ROI projection mask, through considering the position of some ROI on the EPI. By considering the overlap between the fluence and ROI projections, one is able to consider the regions on the EPI for which some structure received dose at any point during the treatment delivery.

Fig. 7.e) and Fig. 7.f) show the correlation between change in D98 and D0.03cc with mean weighted fluence percentage difference for the PTVs and spinal cords, respectively. Pearson's and Spearman's correlation coefficients of 0.69 and 0.72 for the PTVs, and 0.35 and 0.21 for the spinal cords, suggest that a strong correlation exists for D98 and mean weighted percentage difference, and a weak correlation exists with D0.03cc and mean weighted percentage difference. In comparison to the correlation data with the ROI projection masks applied, Pearson's and Spearman's correlation coefficients increase for the cases of the PTV projections and decrease in the case of utilising the spinal cord projections. All correlation coefficients decreased relative to the correlation data with no mask applied, however the sensitivity of the correlation with the intra-angle ROI and fluence projection mask applied significantly increased, as shown by the steeper slopes.
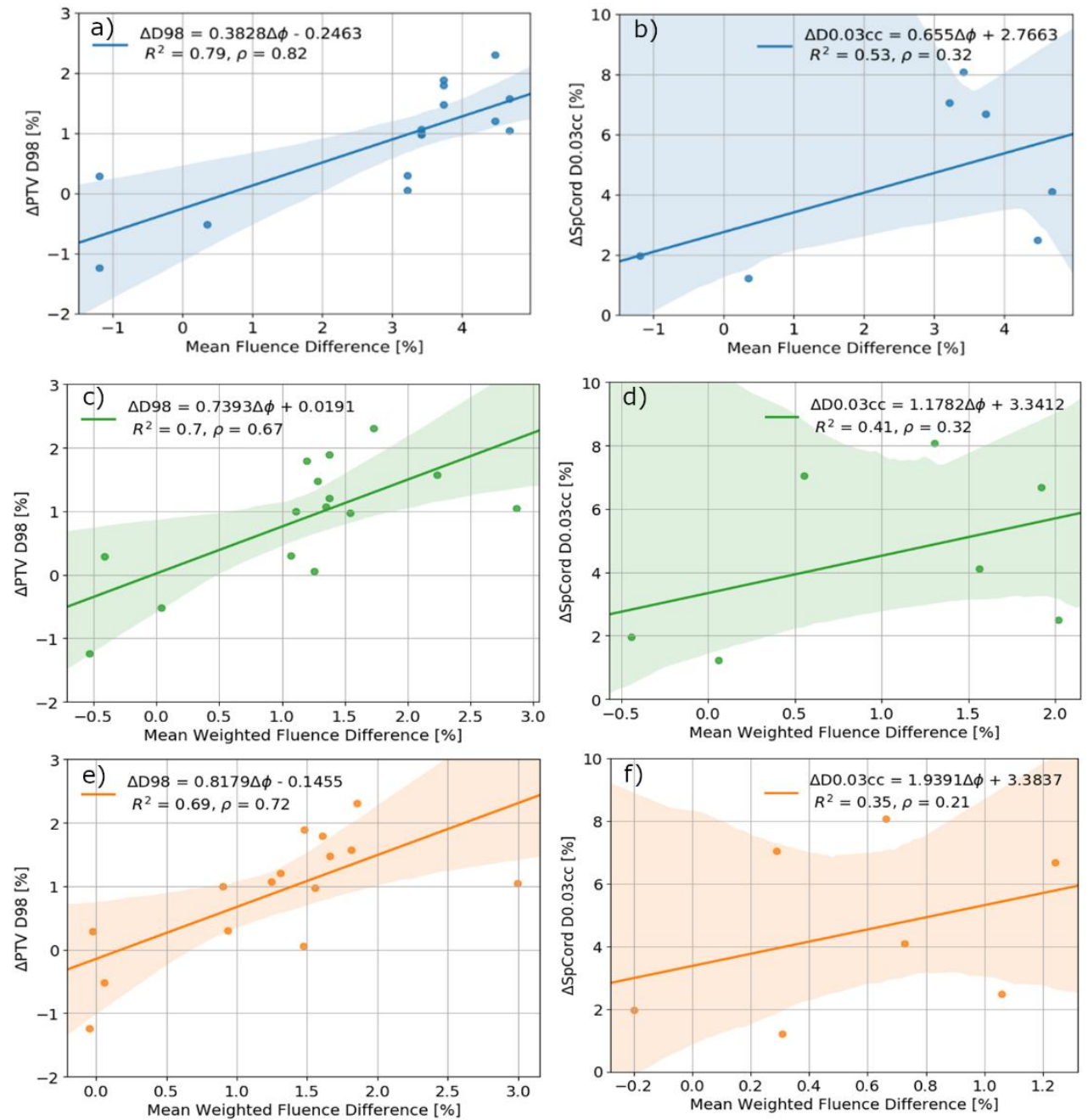

Fig. 7 Correlation between change in D98 and D0.03cc with change in mean fluence percentage difference with no mask applied for a) the PTV(s) and b) spinal cord; with the projection mask applied for c) the PTV(s) and d) spinal cord; and with the intra-angle fluence projection mask applied for e) the PTV(s) and f) spinal cord. 


\subsection{Discussion}

Change in transit fluence, as measured via mean percentage difference, was found to be strongly correlated with change in PTV D98 and moderately correlated with change in spinal cord D0.03cc with no weighting mask applied. Pearson's and Spearman's correlation coefficients were found to decrease when applying a ROI projection mask to the predicted EPIs, however still yielding strong and moderate correlations, respectively. No statistically significant difference between the correlation coefficients was observed between the two sets $\left(p_{P T V}=0.32\right.$ and $p_{\text {SpCord }}=0.41$ ), however the slope of the regression was found to increase by $93 \%$ and $80 \%$ for the PTVs and spinal cords, respectively. Taking the weighting mask through another layer of complexity via an intra-angle convolution with the MLC fluence projections also resulted in no statistically significant difference in the correlation coefficients calculated, relative to no weighting mask applied. The slope of the linear regression fit, however, increased by $113 \%$ and $196 \%$ for the PTVs and spinal cords, respectively; greatly improving the sensitivity of change in transit fluence to a change in key DVH metrics. Future work should consider the specificity of the model, through evaluating some threshold value to trigger the need for plan adaptation, via a receiver operating characteristic (ROC) curve.

This study is consistent with past literature on improving sensitivity via projecting PTVs onto the EPI. For comparison, Piron et al. [6] explored V95\% with mean gamma values across the entire EPI as well as by projecting PTV's. Via a meta-analysis of this study, it was found that the correlation between V95\% and mean gamma increased from $\sim 0.55$ to $\sim 0.70$ when projecting the PTV, as well as the slope of the trend line increasing by $\sim 270 \%$. Overall, projecting the PTVs onto the EPI was found to be beneficial to both the correlation coefficient and sensitivity, which agrees with the results presented.

In this work, patient data was selected on the basis that plan adaptation occurred due to significant morphological changes within the patient, such as tissue shrinkage, resulting in some regions receiving greater than anticipated dose and others lesser. One of the eight patients used for this research, however, was replanned due to significant changes in neck tilt; resulting in poor patient alignment in the clinic. In this scenario, there was poor agreement in the position of PTV's and Spinal Cord between the pCT and rCT and as a result led to unreliable $\Delta D 98$ and $\Delta D 0.03 c c$ values being calculated for the PTV's and spinal cord, respectively. For this reason, the patient data was omitted from this study as the change in datasets reflected an intentional change in patient positioning which would not be encountered when delivering the same plan at different fractions.

When exploring the correlation between change in transit fluence and $\Delta D 0.03 c c$ for the spinal cords, it was observed that a much poorer correlation was found. The sensitivity, however, of the spinal cord DVH metric was drastically improved with the implementation of the weighting mask derived, as demonstrated by the 196\% slope increase in Fig. 7.f) relative to Fig. 7.b). The y-intercept of the linear regressions, however, pose another issue likely due to the small sample size used. Y-intercepts of up to 3.38\% were observed for the spinal cord D0.03cc correlations, suggesting that patients showing a mean (weighted) fluence percentage difference of zero still have some change in dosimetry. Moreover, the 95\% confidence interval of the linear regression fit for all three correlations explored do not include the origin of the graph - suggesting some systematic error is present within the data obtained. In the future, more datapoints, particularly for the spinal cord results, should be obtained to yield more reliable results. A forced y-intercept could also be explored, however addressing the systematic error would be the preferential approach to minimising this issue. 
It is important to note that the DVH metric considered for the spinal cord, D0.03cc, considers the nearmaximum dose received by the spinal cord and is thus derived from very few voxels. The mean percentage difference, on the other hand, considers the entire volume. D0.03cc is thus much more sensitive to slight changes in the position of the structure, and as a result is unlikely to be well correlated with mean percentage difference. The pCT and rCT's utilised are registered as close as possible, however there are still some slight discrepancies in the anatomy between the two - giving rise to somewhat unreliable D0.03cc values. When considering DVH metrics that utilise the entire volume of the structure, such as mean dose and D98, it is reasonable to expect a good correlation. Future work should thus explore the correlation between mean weighted percentage difference and mean dose received by the parotid glands to explore side effects associated with high parotid gland dose from $\mathrm{H} \& \mathrm{~N}$ radiotherapy such as xerostomia.

For the purposes of this study, pCT's were utilised to avoid any uncertainties associated with deformable image registration of the $\mathrm{pCT}$ to $\mathrm{CBCT}$, and dose calculation uncertainty on CBCT. This, however, introduces a sample size limitation, as one patient plan will only yield one data point per adaptation to be used in the correlation study. In the future, the use of CBCT's would improve the overall sample size obtained, where the gradual change in patient anatomy could also be explored. This has the added benefit in that data points early into the treatment plan would likely include fractions which do not necessarily require adaptation, thus enabling us to explore various action threshold values for deciding when to replan. The sensitivity and specificity of each threshold value could be explored through consideration of a receiver operator characteristic (ROC) curve and the area under the curve (AUC). The simulation toolkit developed would ensure that all changes in transit fluence are a direct result of change in patient anatomy, rather than incorrect positioning, and thus still be of use in assessing a critical threshold value. The toolkit can also be applied to multiple regions of interest within a treatment plan, and can be modified to the clinic's specific tolerance values of, for example, dose to the spinal cord or parotid glands for H\&N cancer plans. The intra-angle convolution mask between the ROI and fluence projections showed to drastically improve the sensitivity of any regression fits and is thus deemed to be useful in assessing organs at risk.

\subsection{Conclusion}

The simulation toolkit developed provides a useful method to explore the correlation between change in transit fluence and change in dosimetry for particular regions of interest. The toolkit was capable of predicting change in transit fluence accurately, where a weighting mask allows the user to consider particular regions on the EPID to improve sensitivity. Change in D98 was strongly correlated with change in mean weighted percentage fluence when considering the PTV's, however change in D0.03 cc was only moderately correlated for the spinal cord OARs investigated in this study. 


\section{References}

[1] Brouwer CL, Steenbakkers RJHM, Langendijk JA, Sijtsema NM (2015) Identifying patients who may benefit from adaptive radiotherapy: Does the literature on anatomic and dosimetric changes in head and neck organs at risk during radiotherapy provide information to help?. Radiother. Oncol. 115(3):285-294. https://doi.org/10.1016/j.radonc.2015.05.018.

[2] Castelli J et al. (2015) Impact of head and neck cancer adaptive radiotherapy to spare the parotid glands and decrease the risk of xerostomia. Radiat. Oncol. 10(1). https://doi.org/10.1186/s13014-014-0318-z.

[3] Belshaw L, Agnew CE, Irvine DM, Rooney KP, McGarry CK (2019) Adaptive radiotherapy for head and neck cancer reduces the requirement for rescans during treatment due to spinal cord dose. Radiat. Oncol. 14(1). https://doi.org/10.1186/s13014-019-1400-3.

[4] van Elmpt W, McDermott L, Nijsten S, Wendling M, Lambin P, Mijnheer B (2008) A literature review of electronic portal imaging for radiotherapy dosimetry. Radiother. Oncol. 88(3): 289-309. https://doi.org/10.1016/j.radonc.2008.07.008.

[5] Piron O, Varfalvy N, Archambault L (2015) MO-F-CAMPUS-J-05: Using 2D Relative Gamma Analysis From EPID Image as a Predictor of Plan Deterioration Due to Anatomical Changes. Med. Phys. 42(6). https://doi.org/10.1118/1.4925466.

[6] Piron O, Varfalvy N, Archambault L (2016) EP-1818: Using ROIs projected on EPID as a predictor of plan deterioration due to anatomical changes. Radiother. Oncol. 119(1): S852-S853.

[7] Piron O, Varfalvy N, Archambault L (2018) Establishing action threshold for change in patient anatomy using EPID gamma analysis and PTV coverage for head and neck radiotherapy treatment. Med. Phys. 45(8): 3534-3545. https://doi.org/10.1002/mp.13045.

[8] Cilla S et al. (2016) Initial clinical experience with Epid-based in-vivo dosimetry for VMAT treatments of head-and-neck tumors. Phys. Med. 32(1): 52-58. https://doi.org/10.1016/j.ejmp.2015.09.007.

[9] Lim SB et al. (2019) Investigation of a Novel Decision Support Metric for Head and Neck Adaptive Radiation Therapy Using a Real-Time In Vivo Portal Dosimetry System. Technol. Cancer Res. Treat. https://doi.org/10.1177/1533033819873629.

[10] You SH et al. (2012) Is There a Clinical Benefit to Adaptive Planning During Tomotherapy in Patients with Head and Neck Cancer at Risk for Xerostomia. Am. J. Clin. Oncol. 35(3). https://doi.org/10.1097/COC.0b013e31820dc092.

[11] Panchal A et al. (2019) dicompyler/dicompyler-core v0.5.5. https://doi.org/10.5281/ZENODO.3236628. 


\section{Figures}

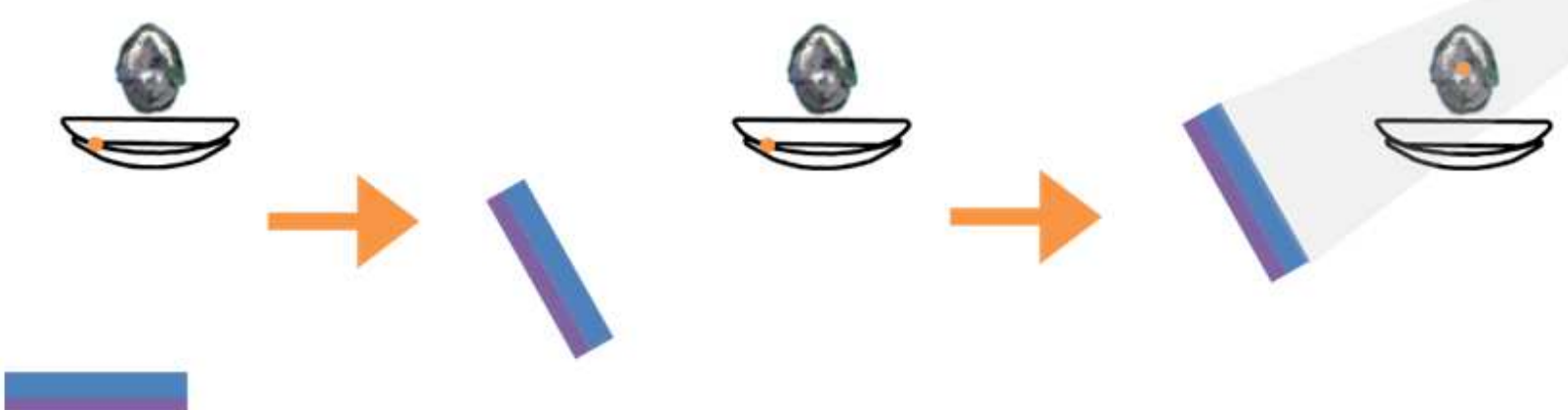

\section{Figure 1}

EPID extraction process repeated along every control point for both the PCT and rCT. EPID first positioned $50.0 \mathrm{~cm}$ below origin, then rotated to a given control point angle, and translated to $50.0 \mathrm{~cm}$ from isocenter


\section{Figure 2}

Process used to derive an intra-angle ROI and fluence projection mask. For each control point, a) the structure projection mask and b) fluence projection is used to obtain d) the overlap between the two, as visualised by c). This process is repeated for each control point to achieve e) the final intra-angle ROI and fluence mask 

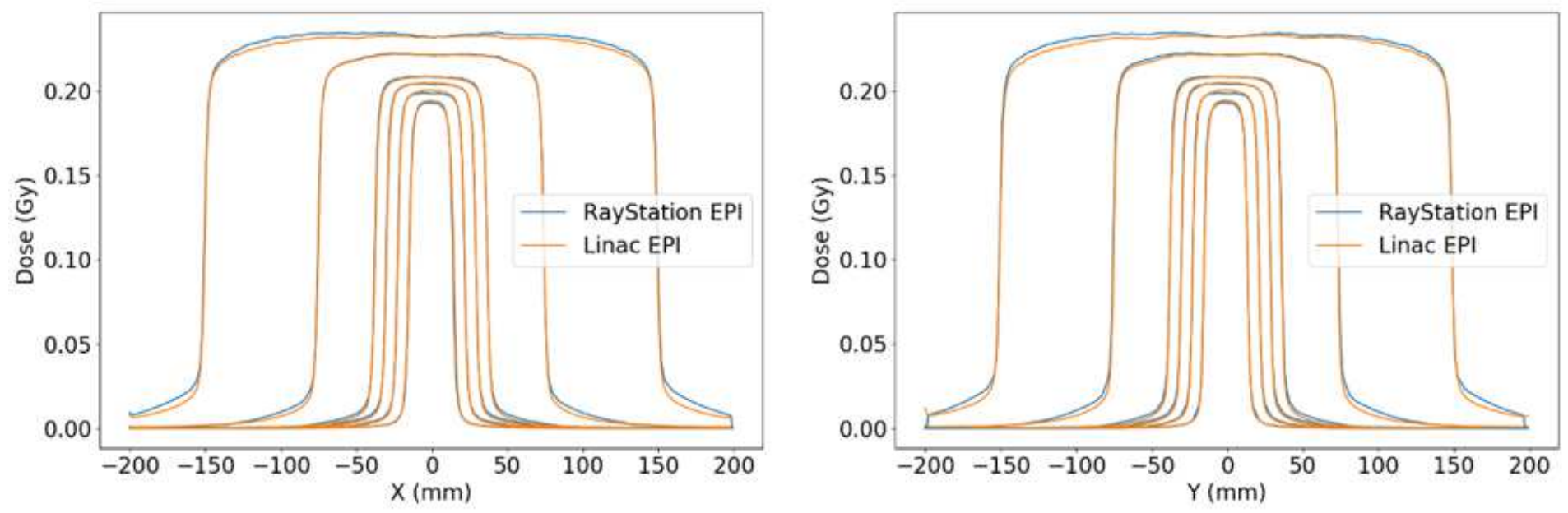

Figure 3

True (Linac) and predicted (RayStation) non-transit beam profiles with EPID positioned at $150 \mathrm{~cm}$

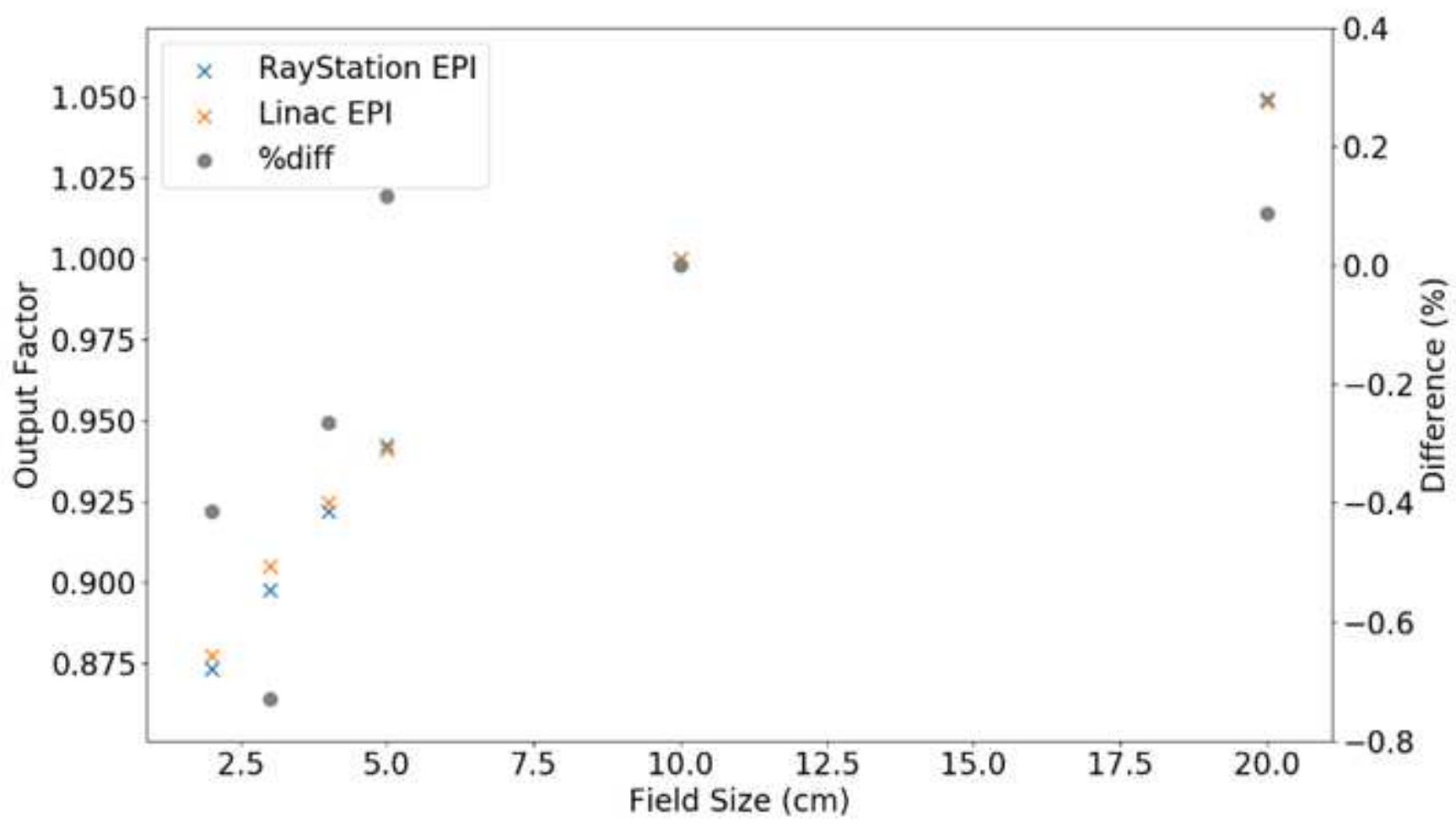

\section{Figure 4}

True (Linac) and calculated (RayStation) output factors from square fields, with percentage difference 

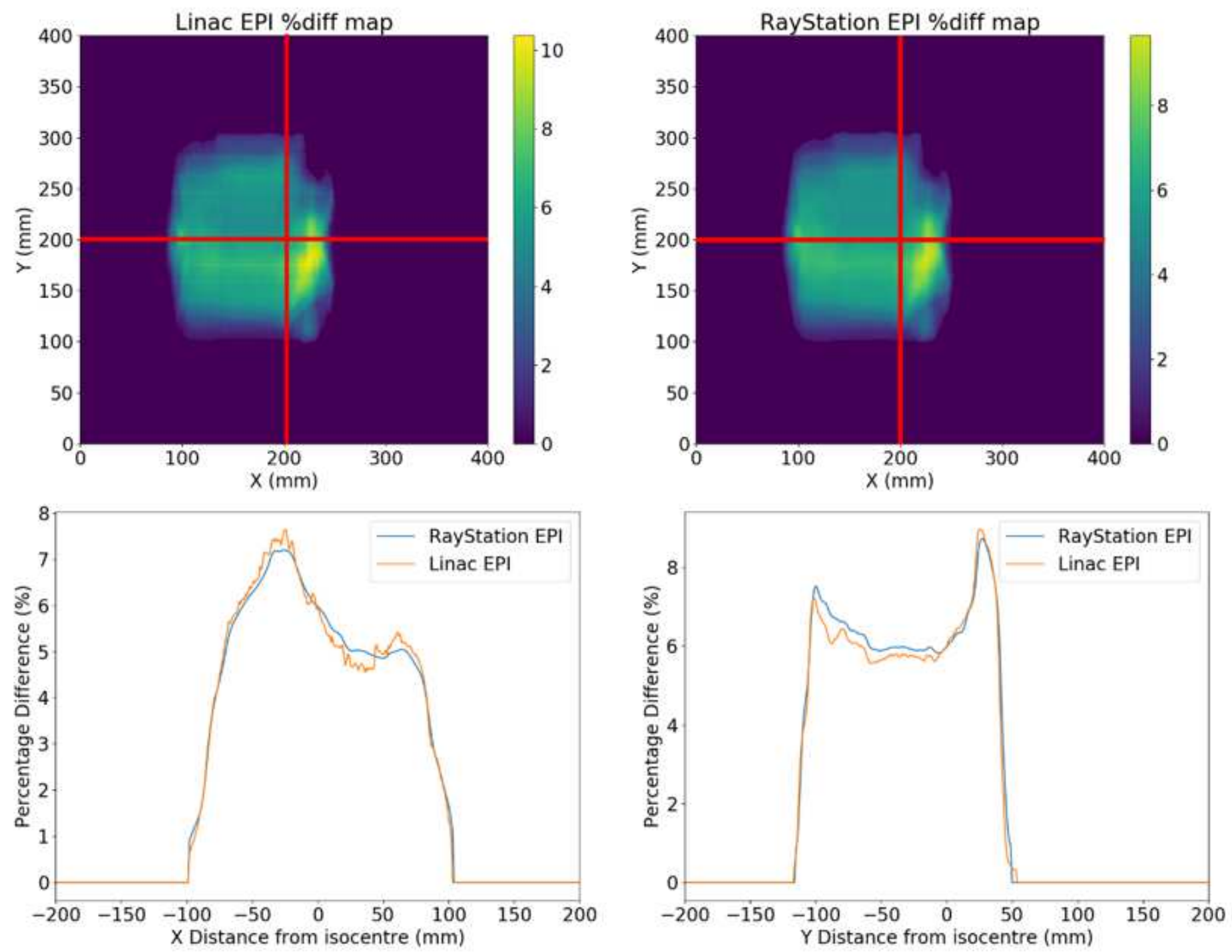

\section{Figure 5}

True (Linac) and calculated (RayStation) 2D percentage difference maps used for model validation, with $x$ and $y$-profiles
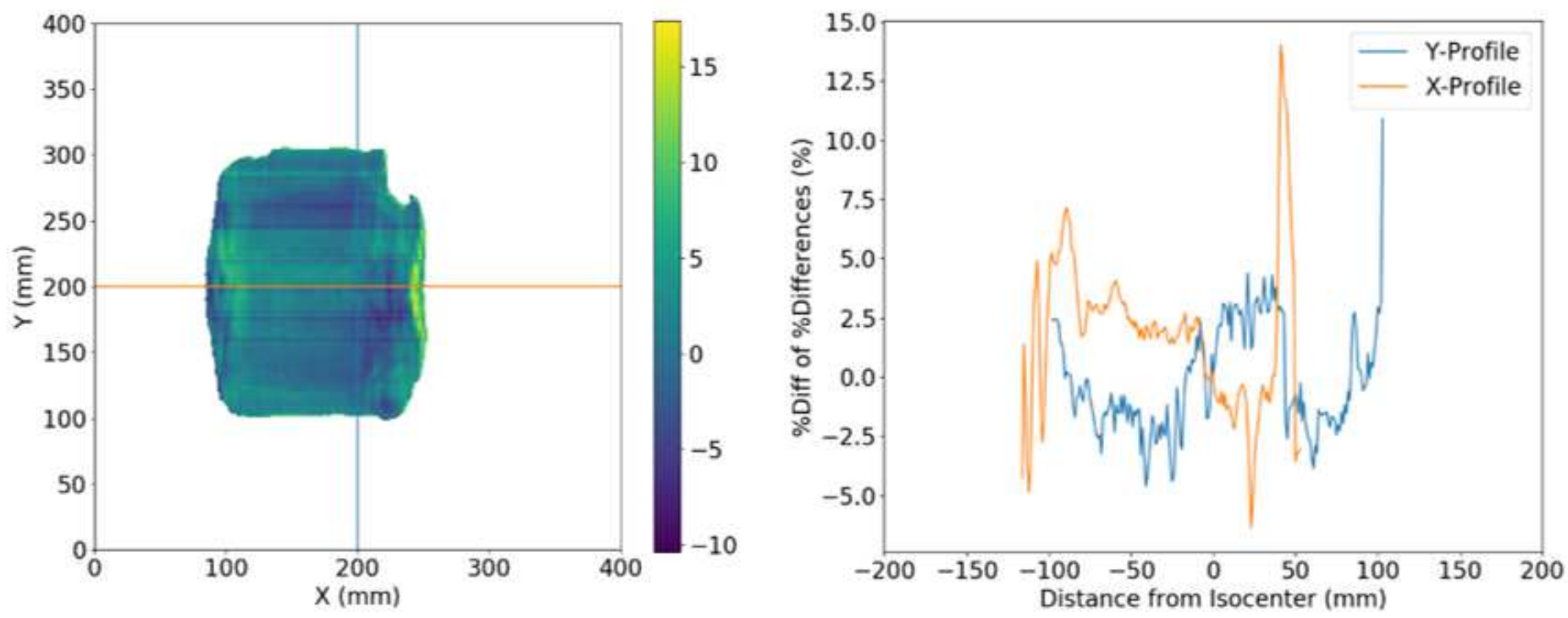
Figure 6

2D percentage error map of the 2D percentage difference maps used for EPID model validation, with Xand Y-profiles


Figure 7 
Correlation between change in D98 and D0.03cc with change in mean fluence percentage difference with no mask applied for a) the PTV(s) and b) spinal cord; with the projection mask applied for $c$ ) the PTV(s) and d) spinal cord; and with the intra-angle fluence projection mask applied for e) the PTV(s) and f) spinal cord. 